\title{
Narrativa
}

\section{Gli studi postcoloniali italiani fuori confine in due diversi approcci}

\section{Franco Manai}

\section{(2) OpenEdition}

1 Journals

\section{Edizione digitale}

URL: https://journals.openedition.org/narrativa/915

DOI: $10.4000 /$ narrativa.915

ISSN: 2804-1224

\section{Editore}

Presses universitaires de Paris Nanterre

\section{Edizione cartacea}

Data di pubblicazione: 1 décembre 2016

Paginazione: 215-225

ISBN: 978-2-84016-266-7

ISSN: 1166-3243

\section{Notizia bibliografica digitale}

Franco Manai, «Gli studi postcoloniali italiani fuori confine in due diversi approcci», Narrativa [Online], 38 | 2016, online dal 01 janvier 2022, consultato il 22 février 2022. URL: http://

journals.openedition.org/narrativa/915 ; DOI: https://doi.org/10.4000/narrativa.915

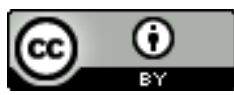

Narrativa est mise à disposition selon les termes de la Licence Creative Commons Attribution 4.0 International. 


\section{Gli studi postcoloniali italiani fuori confine in due diversi approcci}

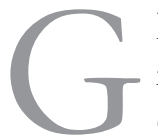

li studi sulla letteratura postcoloniale italiana all'estero sono molto influenzati da quanto avviene all'interno dell'accademia nord-americana che esercita, come è noto, una sorta di predominio e di egemonia sullo sviluppo di molte correnti di studio grazie al controllo di un cospicuo numero di riviste accademiche, di case editrici, di convegni internazionali e di posti universitari. Ci sono alcune modalità che riscuotono particolare successo e determinano conseguenze piuttosto serie. Iniziamo la nostra discussione col volume di Graziella Parati, Migration Italy. The art of talking back in a destination culture $^{1}$. Si tratta di un libro importante negli studi postcoloniali italiani in quanto sorta di summa teorica del pensiero dell'autrice che già dai primi anni ' 90 si era interessata alla letteratura prodotta in italiano da immigrati e aveva svolto, dalla sua postazione accademica statunitense, attività pionieristica di promozione e di diffusione sia della produzione artistica migrante sia degli studi culturali e postcoloniali in Italia ${ }^{2}$. Anche dopo il volume preso in considerazione in queste pagine, Parati ha continuato a pubblicare sull'argomento della migrazione ${ }^{3}$ e a

1. Parati, Graziella, Migration Italy. The art of Talking Back in a Destination Culture, Toronto, University of Toronto Press, 2005.

2. Parati, Graziella, 'When the 'Other' is Black: Portraits of Africans by Contemporary Italian Writers”, Romance Languages Annual, n. 7, 1993, pp. 272-277; Public history, private stories Italian women's autobiography, Minneapolis, University of Minnesota Press, 1996; "Strangers in Paradise: Foreigners and Shadows in Italian Literature", in AlLeN, Beverly, Russo, Mary (a cura di), Revisioning Italy. National Identity and Global Culture, Minneapolis-London, University of Minnesota Press, 1997, pp. 169-190; Mediterranean Crossroads: Migration Literature in Italy, Madison [N.J.], Fairleigh Dickinson University Press, 1999.

3. PARATi, Graziella, "Comunità, diritti umani e testi multiculturali”, in QuaQuarelLI, Lucia (a cura di), Certi confini. Sulla letteratura italiana dell'immigrazione, Milano, Morellini, 2010, pp. 23-41; "Introduction", in PIVETTA, Oreste (a cura di), I Was an Elephant Salesman. Adventures between Dakar, Paris, Bloomington and Indianapolis, Indiana Univer- 
essere riconosciuta, anche nelle sintesi di storia della critica sull'argomento ${ }^{4}$, come un'autorità per così dire teorica e pratica, assieme al critico italiano Armando Gnisci, studioso anarchico da lei tanto differente dal punto di vista ideologico ${ }^{5}$.

Graziella Parati incentra la sua ricerca sulla letteratura migrante intorno ai concetti di alterità e di ibridazione e non si sottrae alla nuova tendenza personalizzante per la quale la critica deve definire il luogo dove si colloca e il contesto da cui prende le mosse per formulare le sue teorie. Ciò intende non come luogo teorico o costellazione ideologica, che non è più di moda negli Stati Uniti, ma come condizione psicosociologica e bagaglio di esperienze di vita individuali. Il lavoro della critica viene inquadrato in una cornice di vissuti, emozioni, ricordi, saperi e desideri personali, che è importante e anche illuminante, ma non basta a consolare il lettore per la mancanza di un quadro di riferimento ideale e generale, mancanza che si sente lungo tutto il percorso del libro. La scrittrice ci informa sulla propria famiglia di origine, la quale mostra una formazione abbastanza comune nell'Italia del boom economico degli anni ' 60 , in cui l'accelerata urbanizzazione metteva in contatto persone provenienti da province e dialetti diversi, creando un'omogeneità linguistica e culturale sul cui carattere fittizio o reale si potrebbe discutere. Ricostruendo a posteriori la sua esperienza, l'autrice definisce come identità ibride quelle dei genitori, nonni e parenti in genere e certamente anche quelle della sua generazione, che conserva in sé le diverse radici degli antenati. In seguito al suo trasferimento per studio e poi per lavoro negli Stati Uniti, la condizione di ibridazione culturale e psicologica si complica ulteriormente anche in conseguenza della caratteristica nomade di questi trasferimenti. Infatti non si tratta di un viaggio di andata e ritorno, ma di un movimento pendolare che mantiene attuali tutte le appartenenze e non permette il superamento di nessuna. $\mathrm{E}$ da questa situazione di condivisione personale dell'esperienza nomade e dell'individualità ibrida, l'autrice si sente autorizzata a studiare gli agenti della migrazione e le loro fatiche letterarie non come un out-

sity Press, 2010, pp. XI-XV; Parati, Graziella, Tamburri, Anthony Julian, The Cultures of Italian Migration: Diverse Trajectories and Discrete Perspectives, Madison [N.J.], Fairleigh Dickinson University Press, 2011.

4. Mengozzi, Chiara, Narrazioni contese. Vent'anni di scritture italiane della migrazione, Roma, Carocci, 2013.

5. GNISCI, Armando. Il rovescio del gioco, Roma, Sovera multimedia, 1993; La letteratura italiana della migrazione, Roma, Lilith, 1998; Via della decolonizzazione europea, Isernia, Cosmo Iannone, 2004; Esercizi italiani di anticolonialismo, Roma, Edizioni Efesto, 2016; (a cura di), Nuovo Planetario Italiano. Geografia e antologia della letteratura della migrazione in Italia e in Europa, Troina, Città Aperta, 2006. 
sider ma quasi dall'interno, fatta salva la condizione privilegiata che contraddistingue la propria migrazione, quella cioè di intellettuale bianca, occidentale con disponibilità economica e libertà di movimento nel globo terrestre. Forse è proprio a partire da questo particolare apparentemente insignificante che alcuni punti nebulosi della sua indagine possono essere spiegati.

Lo scopo del libro va al di là dell'analisi della critica letteraria ed è quello di creare un linguaggio nuovo, sia per parlare della migrazione senza dimenticare la natura dialogica dell'identità secondo Taylor e Appiah ${ }^{6}$, sia per ridefinire l'alterità. Non è chiaro quale necessità spinga quest’impresa, né in base a che cosa sia necessario ridefinire l'alterità. In che cosa il linguaggio vecchio è insufficiente per parlare della migrazione e cosa giustifica la scelta a priori di considerare l'identità una variabile relazionale secondo l'antiessenzialismo militante di Taylor e quello ancora più estremo di Appiah? Il discorso sull'identità è oggi uno dei più sfruttati nel mondo dalle propagande nazionalistiche di tutti i tipi e di ogni tendenza politica, quindi un concetto pericoloso da utilizzare e difficile da analizzare e teorizzare. Quando il Cardinale Biffi, come riporta Parati, dà esempio di tipica chiusura mentale contro l'immigrazione e afferma che bisogna ammettere in Italia solo immigrati cattolici per preservare l'identità nazionale, è chiaro che sta parlando del potere della Chiesa Cattolica in Italia, nel senso che è quello che bisogna preservare. In questo caso la funzione manipolatoria dell'identità nazionale è estremamente evidente. Secondo i fatti delle cronache degli ultimi duemila anni, le religioni si prestano magnificamente alla manipolazione, solo che in altri tempi non si parlava di identità religiosa ma di fede, di valori, di leggi e di tutto quello che doveva essere difeso senza esclusione di mezzi. Oggi identità nazionale è la parola d'ordine che viene sbandierata contro l'invasione dei migranti, come anche per spingere i popoli verso guerre di cui non hanno nessun bisogno e non sentono nessun desiderio. Parati si propone di smontare storicamente il concetto di identità nazionale nel caso dell'Italia dimostrando che quell'omogeneità identitaria e culturale percepita non corrisponde a una realtà oggettiva. Infatti l'Italia è stata sottoposta, a partire dall'inizio del Novecento - ma Parati si concentra soprattutto sul secondo dopoguerra - a un processo di omogeneizzazione forzato attraverso l'imposizione di una lingua comune, l'italiano standard della scuola dell'obbligo e

6. Taylor, Charles, "The Politics of Recognition", in Hargreaves, Alec (a cura di), Multiculturalism: Examining the Politics of Recognition, Princeton, Princeton University Press, 1994, pp. 27-73; Appiah, Anthony, "Identiy, Autenticity, Survival: Multicultural and Social Reproduction", in GutMann, Amy (a cura di), Multiculturalism: Examining the Politics of Recognition, Princeton, Princeton University Press, 1994, pp. 149-63. 
dell'amministrazione pubblica. Sono stati negati e relegati nel privato e nell'irrilevanza i numerosi dialetti e le lingue regionali, cancellando così in gran parte la varietà di culture mediterranee che formano la cultura italiana, o per lo meno ignorandole e rendendole invisibili. La cultura italiana è quindi una pluralità di culture e la lingua italiana una pluralità di lingue la cui espressione e il cui sviluppo sono stati repressi per permettere di creare l'illusione di una cultura omogenea, utile a una coesione di cittadini in una nazione dal potere accentratore, funzionale a una governabilità e a una malleabilità delle masse così inquadrate.

Tuttavia l'omogeneità creata dalla scolarizzazione di massa e, dagli anni '50 in poi anche dai media, cinema, radio, televisione su scala nazionale, è veramente solo fittizia? Il fatto che sia stata imposta non la rende meno reale. Parati stessa cita Étienne Balibar ${ }^{7}$, secondo cui ogni comunità sociale riprodotta attraverso le istituzioni è immaginaria. Da ciò risulta che solo le comunità immaginarie sono reali. La comunità e l'identità percepite sono anche le uniche reali, non quelle che lo storico ricostruisce a tavolino. Qual è dunque l'utilità di una rivisitazione storica fatta al fine di rivelare l'artificialità dell'unità culturale, linguistica e letteraria italiana? Parati sembra attribuire a questa dimostrazione more geometrico una grande importanza nell'ambito della relazione degli italiani, non più ancorati alla loro artificiale identità, con gli immigrati, la cui diversità non apparirà più tanto grave in mezzo a tante diversità riscoperte, per esempio tra milanesi, comaschi, ecc. Parati si ripropone di investigare le politiche culturali italiane che hanno manipolato i concetti di uguale e diverso producendo concetti ad hoc di italianità e di estraneità. Cita Alessandro Dal Lago che ha articolato il concetto di "essere una non persona" e afferma che un migrante senza documenti è un membro produttivo della comunità ma può essere espulso in qualsiasi momento ${ }^{8}$. Ora questo è indubbiamente vero, ma ha poco a che fare con le politiche culturali italiane e molto invece con la struttura politica dello stato nazione e della sua storia nel XX secolo, come afferma Hannah Arendt in un testo del 1951, Le origini del totalitarismo ${ }^{9}$, definendo la situazione di apolide come l'espressione e il fenomeno politico più caratteristico del XX secolo. Per fondare la nazione, su cui lo stato basa la sua legittimità, è necessario mondarla dell'eterogeneità, a meno che non sia necessario un certo controllato pluralismo per mantenere

7. Balibar, Étienne, "The Nation Form: History and Ideology", in Balibar, Étienne, Wallerstein, Immanuel (a cura di), Race, Nation, Class: Ambiguous Identities, New York, The Feminist Press, 1999, pp. 86-106.

8. Dal Lago, Alessandro, Non persone: l'esclusione dei migranti in una società globale, Milano, Feltrinelli, 1999.

9. ARendt, Hannah, Le origini del totalitarismo [1951], Torino, Einaudi, 2009. 
insieme la sua omogeneità. Si tratta di un problema ben più profondo e strutturale delle caldane identitarie sempre manipolate. A questo proposito Judith Butler suggerisce che sia venuto il momento di sviluppare forme post-nazionali di opposizione politica ${ }^{10}$. Tuttavia il problema è complesso e Gayatry Spivak, nello stesso volume, nota che il declino degli stati nazionali oggi sta avvenendo come effetto della globalizzazione, della subordinazione economica e politica al capitale globale. Di conseguenza il riaffiorare della comunità multietnica, che covava sotto la cenere dell'unità nazionale, rischia di fare il gioco di quel potere nascosto dietro mille nomi e mille tentacoli che è il capitale internazionale, su cui Hannah Arendt non arrivava a riflettere. Lo stato nazionale oggi è una struttura astratta e minima che dobbiamo difendere, sostiene Spivak, perché è il nostro alleato, deve diventare lo strumento della ridistribuzione. Se da un lato è quindi possibile ripensare la struttura dello stato nazionale e magari addirittura tornare ai regionalismi, come auspica Butler, è importante non sottovalutare il pericolo di vedersi poi in balia di un potere senza centro ma ugualmente totalizzante. L'interpretazione della globalizzazione dal basso che Parati difende è quella che permette all'individuo di ri-scriversi. Siamo sempre nell'ambito della letteratura: scriversi, ricollocarsi nello spettro globale, imbastendosi un'identità ibrida e variegata, intrisa di non appartenenze e in processo di continua rottura con l'esistente. Questa globalizzazione dal basso, purtroppo, questa atomizzazione dell'umano, dell'individuo che da solo si cerca un posticino al sole nella non società indeterminata ed entropica, non solo è spaventosa in sé, ma lo è di più quando perde di vista l'insieme, il sistema, il quadro generale e le sue leggi, che sì esistono e all'insaputa dell'individuo lo dominano.

Parati vede nella risposta contestataria al monoculturalismo nazionalista una via per fare emergere le nuove individualità ibride, anche se ammette che si tratta di una via debole. La letteratura postcoloniale italiana e italomigrante costituirebbe questa risposta, ma, aggiungiamo noi, essa non è esente dalle manipolazioni, anche perché spesso non ha altra scelta che scendere a compromessi ed entrare in complicità con l'apparato che vorrebbe smontare, perché solo in esso gli immigrati si possono scavare una nicchia in cui mantenere qualcosa della loro identità e diversità. Sebbene, come dice Spivak, le culture della diaspora siano molto diverse da quelle dei paesi d'origine, e sebbene siano già di per sé ibride, l'entrata delle scritture migranti nella letteratura italiana ha provocato una trasformazione, una rottura della superficie in tinta unita di quella

10. Butler, Judith, SpIVAK, Gayatri Chakravorty, Who Sings the Nation-State. Nation, Politics, Belonging, London-New York, Seagull Books, 2007. 
produzione letteraria, una ricolorazione della cultura, facendo emergere le differenze in essa già esistenti e imponendo la presenza di nuove differenze che avviano processi di ibridazione nuovi. La domanda se i testi degli scrittori migranti siano vera letteratura o fenomeni di rilevanza sociologica può trovare risposta, secondo Parati, in una definizione di letteratura come quella di Maurizio Viano, critico anche lui dell'accademia statunitense, paladino della political correctness: "Al di là delle categorie del bello o del brutto, il testo letterario richiama con forza la nostra attenzione per la sua capacità di costringerci a confrontarci con questioni di urgenza incomparabile" 11 . Parati individua queste questioni "di urgenza incomparabile", nel "futuro delle letterature in italiano, [nel] futuro delle culture italiane, e [nel rapporto tra le culture locali e quelle globali considerato alla luce delle migrazioni da e verso l'Italia" ${ }^{\prime 2}$.

Qualsiasi cosa questo voglia dire, sembrerebbe di trovarsi comunque di fronte a una definizione tautologica: un testo è letterario in quanto ci confronta con problemi di letteratura. Parati non accetta, nonostante non le sia possibile appoggiare con argomenti solidi questa posizione, di relegare la letteratura migrante agli studi sociali, perché vorrebbe arrivare a teorizzare la possibilità di una letteratura come luogo aperto al cambiamento, luogo di intertestualità in cui si verificano processi di ibridazione. Il passato culturale italiano interseca, secondo lei, un presente multiculturale che si proietta in un contesto europeo di culture ibride. Ora, se è vero che tutte le culture umane conosciute sono ibride, come riconosceva anche il padre di tutti gli strutturalismi, Claude Lévi-Strauss in Race et histoire (1952), è anche vero che, per quanto prendano le loro componenti da fonti diverse, esse tendono a darsi una struttura coerente in cui gli elementi si tengono insieme: questa è la vita che c'è in loro. In un'orgia di ibridazione, dove tutti si ibridano con tutti, finisce per perdersi anche il concetto di cultura stessa e di comunità umana. Nella prospettiva di Parati, l'ibridazione appare il massimo bene, la via e la meta, il contenuto e la forma. La letteratura in quanto veicolo di ibridazione culturale compie il suo destino.

La positionality di cui si diceva all'inizio si rivela infine ingenua figlia della grande pantomima del politically correct, quella patina di rispetto dei valori la cui consistenza e presa sulla realtà si mostrano in tutta la loro ipocrita evanescenza nei momenti cruciali della storia, per esempio nel 2016 negli Stati Uniti con la

11. Viano, Maurizio, "Ecce Foemina”, Annali d'Italianistica, n. 4, 1986, pp. 223-241 (p. 224).

12. Parati, Graziella, Migration Italy, cit., p. 63 (traduzione nostra di "the future of literatures in Italian, the future of Italian cultures, and the relationship between local and global cultures seen under the light of migration from and to Italy"). 
spudorata ascesa di Donald Trump per le elezioni presidenziali, in Europa con l'implosione di fronte all'attacco dei migranti, nel "Mondo" orientale con le posizioni espresse dal Dalai Lama, per anni nume indiscusso e punto di riferimento per il buonismo internazionale: "La Germania non può essere un paese arabo [... i migranti] stanno diventando troppi", ha detto il leader spirituale tibetano immediatamente sostenuto dai media della finanza internazionale ${ }^{13}$. Dopo anni di orge di politically correct, a sinistra, a destra, al centro, salta fuori che negli Stati Uniti gli elettori sono capaci di votare in massa, nientemeno che per la presidenza, un personaggio che è politicamente scorretto a parole e scorrettissimo di fatto, a cominciare dal modo di trattare con le donne, per non dire dei migranti musulmani o messicani che siano. Tutto questo significa che i politically correct americani, quando si sentono per qualche motivo messi alle strette, ignorano la political correctness, oltre che in sostanza, come sempre hanno fatto, anche nella forma. E per l'Europa l'inconsistenza della finzione ipocrita è anche più evidente, col ripristino delle frontiere, con buona pace di Schengen e accordi vari sulla libera circolazione e "volemose" bene di tutti i tipi. Vorremmo precisare, per scansare degli equivoci, che non intendiamo assolutamente dire che se gli americani voteranno il filosofo dell' Art of the Deal, la responsabilità è dei politically correct. Vogliamo semplicemente significare che l'atteggiamento politically correct non è garanzia di correttezza di approccio critico.

Sembra infatti che la posizione di migrante privilegiata e soprattutto l'esperienza di ibridazione felice di Parati stinga nell'analisi e funga quasi da prototipo di quel che viene presentato come meta e scopo della letteratura migrante. Se si deve gratitudine all'onestà intellettuale dell'autrice, non si può comunque condividerne la visione.

Non tutti gli italianisti che operano fuori d'Italia si uniformano alla vulgata dell'ibridazione a tutti i costi. Molto diversa da quella che abbiamo visto è l'impostazione delle ricerche di Silvia Contarini che opera all'Université Paris Nanterre. Le affiliazioni politiche di Contarini non sono un mistero e appaiono chiare nella sua narrativa: Noi veri delinquenti ${ }^{14}$ è il titolo del suo romanzo che riprende uno slogan antifrastico della sinistra extraparlamentare dell'Italia degli anni '70 "Gui e Tanassi sono innocenti, siamo noi i veri delinquenti". Ci limiteremo a pochissimi esempi che crediamo abbastanza significativi. Nel saggio

13. Spineldi Barrile, Andrea, "Il Dalai Lama sui rifugiati in Europa: cosa ha detto veramente", International Business Times, 1 giugno 2016, http://it.ibtimes.com/ il-dalai-lama-sui-rifugiati-europa-cosa-ha-detto-veramente-1453541.

14. Contarini, Silvia, Noi veri delinquenti, Roma, Fazi, 2005. 
"Narrazioni migrazioni e genere"15, la studiosa attraverso un'approfondita analisi di testi di numerose scrittrici migranti, rileva come dalle scritture al femminile emerga con insistenza il sovrappiù di peso che grava sulle spalle delle donne migranti rispetto a quello che grava sulle spalle degli uomini. Le donne infatti si trovano a essere dilacerate da due tendenze opposte. Su di esse incombe in primo luogo il compito di tramandare le radici, di perpetuare di generazione in generazione l'attaccamento a ciò che si è potuto portare con sé dalla terra d'origine. Si tratta spesso di teorie e pratiche fortemente oppressive e limitanti nei confronti delle donne, ma che proprio le donne, per motivi complessi che non è il caso di affrontare in questa sede, si assumono la responsabilità di perpetuare. In senso opposto agisce un'altra esigenza, che pure riposa in capo alle donne, quella di trovare una qualche conciliazione, un qualche compromesso con la società ospitante che consenta, se non l'integrazione, per lo meno la sopravvivenza. Sono le donne infatti che intessono rapporti di vicinato, che vanno a fare la spesa, che trattano con i padroni di casa, che, in una parola, assicurano, in contesti anche di grande ostilità, quella rete di rapporti sociali senza la quale non sarebbe possibile la stessa permanenza delle famiglie di migranti nei paesi di arrivo. La compresenza di queste due spinte intimamente contraddittorie si risolve in molti casi in una palese celebrazione del compromesso. Pur con tante differenze, la missione che più di frequente le protagoniste di queste storie fanno propria, è quella di operare un'attenta selezione volta a conservare quanto possibile della cultura di provenienza e ad accogliere quel che nella cultura di arrivo risulti o accettabile o inevitabile.

Tutto ciò comporta, in queste scritture, la tendenza a silenziare quanto di negativo comunque esiste nella cultura di partenza, tacendo o trasfigurando le pratiche e i modi di vita che, soprattutto agli occhi occidentali, risulterebbero, e di fatto risultano, più arretrati e inaccettabili. Su un altro versante una grande attenzione è posta a mettere tra parentesi gli aspetti più negativi della vita dell'emigrato in occidente, e soprattutto dell'emigrata. Contarini rileva come in questi romanzi l'attenzione si concentri in genere su donne migranti, ma emancipate, donne che studiano e che lavorano, che sanno conquistarsi il loro posto nel mondo al pari, se non di più, delle consorelle italiane. Bassissima è la percentuale di collaboratrici domestiche e soprattutto di prostitute. Questo tipo di personaggi compare, ma quasi sempre ai margini della narrazione. Particolarmente significative ci sembrano le pagine che Contarini dedica a Madre piccola di

15. Contarini, Silvia, "Narrazioni migrazioni e genere", in Quaquareldi, Lucia (a cura di), Certi confini, cit., pp. 119-159. 
Cristina Ali Farah ${ }^{16}$. La protagonista racconta come grazie all'opposizione dei suoi genitori ella sia sfuggita, con grande dispetto del resto della famiglia, all'infibulazione. Di ciò è lietissima e manifesta tutta la sua gratitudine verso chi l'ha sottratta a quella mutilazione. Tuttavia la scena stessa della cerimonia alla quale hanno partecipato le cugine è raccontata con molta circospezione: viene rievocata un'atmosfera ovattata, piena di liturgia, di mistero e di suggestione, con la rappresentazione dell'ingenuo orgoglio che manifestano le bambine infibulate rispetto alla delusione e all'invidia che le escluse provano verso le reginette della festa. È questo sicuramente un modo di dire e non dire, raccontare e travisare, denunciare ed edulcorare.

Contarini osserva che il quadro che emerge dall'insieme di queste narrazioni è molto diverso da quello ben più crudo che salta fuori dalle indagini e dalle ricerche sociologiche le quali dimostrano come il destino di larga parte delle donne migranti sia legato alle professioni di cura (badanti e colf) e alla prostituzione. Neanche altri tratti evidenziati dalla ricerca sociologica trovano spesso cittadinanza in questo tipo di narrazioni, come per esempio la diversa dinamicità e intraprendenza dimostrata dalle donne provenienti da paesi africani rispetto ai compatrioti uomini. Piegandosi a svolgere lavori rifiutati dalle autoctone, le donne migrate comunque lavorano; nonostante lo sfruttamento cui sono sottoposte in primo luogo dai loro uomini vicini e lontani, che non lavorano, esse riescono spesso ad accumulare somme di denaro che giungono persino a investire. Per molte donne dunque, come per le senegalesi cui si fa cenno, la vita vissuta nei paesi di destinazione, per quanto dura e combattuta, si risolve in fin dei conti nella conquista di una situazione migliore, di una sostanziale autonomia che sarebbe stata impensabile nel paese d'origine. Registrare fatti come questo metterebbe forse in luce con troppa evidenza le fratture di genere all'interno stesso delle comunità migranti, in aggiunta a quelle di razza e di classe che pure in molte di queste narrazioni, secondo Contarini, si tende a sfumare.

Non mancano però i casi, pochi, di autrici che dimostrano una diversa predisposizione. Interessante l'esempio portato da Contarini dellitalo-indiana Gabriella Kuruvilla. In È la vita dolcezz $a^{17}$ ci imbattiamo in un mondo scisso e frantumato di coppie miste e separate, donne sole, donne abbandonate, figli abbandonati, madri impazzite, prostitute, colf. È un mondo rappresentato con asciutto realismo, senza filtri edulcoranti, al di là di ogni intento conciliatorio e

16. Ali Farah, Cristina, Madre piccola, Milano, Frassinelli, 2007.

17. Kuruvilla, Gabriella, Ė la vita dolcezza, Milano, Baldini Castoldi Dalai, 2008. 
consolatorio. Resta nella memoria il caso dell'immigrata Mina, che a Milano si guadagna la vita tessendo sciarpe di seta poi spacciate nelle boutiques come autenticamente indiane. Un designer con la fissazione dell'India la sposa per ricostruirsi in casa una piccola India portatile e la povera Mina si adatta a vivere e mangiare all'indiana quando avrebbe preferito vivere e soprattutto mangiare all'italiana. La nascita di una coppia di gemelli fa poi saltare il piatto: nel designer si affievolisce la passione per l'esotico orientale ed egli sostituisce la madre dei suoi figli con una giovane bionda americana. Mina non ha preoccupazioni economiche. Il designer da questo punto di vista fa largamente fronte ai suoi doveri. E tuttavia qualcosa si è spezzato. La mattina, in jeans e camicetta Mina porta i figli a scuola, poi torna a casa. Indossa il sari, si raccoglie i capelli, si colora la tikeka sulla fronte, si appende al collo una Barbie e va in giro per Milano gridando "Troia di una Barbie". Nel pomeriggio, dopo aver di nuovo indossato jeans e camicetta e con la fronte pulita, va a prendere i figli a scuola. Come ci invita a pensare Contarini, non si potrebbe forse dare rappresentazione più nitida di un corto circuito umano segnato dalle contrapposizioni di razza, di genere, di classe.

In altri due lavori sulla letteratura migrante, "Ricomposizioni ereditarie: genere, etnia, classe in La straniera di Younis Tawfik" e "Lingue, dialetti, identità. Letteratura dell'immigrazione" ${ }^{18}$, la studiosa applica lo stesso approccio che abbiamo appena delineato, nel primo alla lettura del romanzo dello scrittore iracheno Tawfik ${ }^{19}$, immigrato in Italia nel 1979, e nel secondo, che è un saggio di più ampio respiro, alla questione della lingua rinata, per così dire, in chiave identitaria, con la comparsa della nuova letteratura dell'immigrazione. La puntuale analisi, anche linguistica, di Contarini ci conferma che assegnare alla letteratura un valore d'uso, considerarla alla stregua di uno strumento di docile attuazione di programmi decisi a tavolino nei campus universitari, negli uffici municipali o nei corridoi governativi, comporta sempre il rischio di favorire la diffusione, e in effetti addirittura la nascita, di opere che altro non sono che la

18. Contarini, Silvia, "Lingue, dialetti e identità. Letteratura dell'immigrazione", in Bonnet, Nicolas (a cura di), Particularismes et identités régionales dans la littérature italienne contemporaine, Université de Bourgogne, n. 4, juin 2011, http://revuesshs.u-bourgogne.fr/individu\&nation/document.php?id=559; EAD. "Ricomposizioni identitarie: genere, etnia e classe in La straniera di Younis Tawfik", in FrIED, Ilona (a cura di), Identità italiana e civiltà globale all'inižio del ventunesimo secolo, Budapest, Ponte Alapitvany, 2012, p. 243-254.

19. TAwFik, Younis, La straniera, Milano, Bompiani, 1999. 
proiezione di velleità ideologiche, di malintese buone intenzioni, di perniciose distorsioni del reale.

Le scrittrici e gli scrittori migranti che si riconoscono tali, italiani e non, residenti o clandestini, postcoloniali o meno, meritano rispetto, meritano di essere presi davvero in considerazione per quel che hanno da dire e che effettivamente dicono, per il contributo da loro apportato all'universo della letteratura femminile e maschile, migrante e non migrante, alla letteratura senza aggettivi, quella che ai nostri occhi curiosi e ansiosi offre il prisma attraverso cui riusciamo a vedere forse non la Verità, ma magari almeno ciò che non siamo, ciò che non vogliamo.

Franco MANAI

The University of Auckland 
